\title{
Rhizosphere effect on removal and bioavailability of PAHs in contaminated agricultural soil
}

\author{
$\mathrm{Na} \mathrm{Li}^{1} \cdot$ Katarzyna Możdżeń ${ }^{2} \cdot$ Zhiguo Zhang $^{1} \cdot$ Chong Liu $^{1}$ • Peiman Zandi ${ }^{1}$. Muhammad Fahad Sardar ${ }^{1}$. \\ Changxiong Zhu ${ }^{1}$
}

Received: 29 July 2019 / Accepted: 10 January 2021 / Published online: 11 March 2021

(C) The Author(s) 2021

\begin{abstract}
The rhizosphere effect of ryegrass (Lolium perenne L.) on polycyclic aromatic hydrocarbons (PAHs) dissipation, bioavailability and the structure change of microbial community was investigated using a compartmented device-rhizobox. The PAHs removal efficiency, bioavailability and the change in structure of the microbial community were ascertained using HPLC, Tenax-TA extraction and PCR-DGGE, respectively. The results showed that in the root area (R1) and bulk soil (CK), the removal of 3-ring PAHs were $97.72 \pm 0.34 \%$ and $95.51 \pm 0.75 \%$, 4-ring PAHs were $89.01 \pm 1.61 \%$ and $78.65 \pm 0.47 \%, 5$-ring PAHs were $77.64 \pm$ $4.05 \%$ and $48.63 \pm 3.19 \%$, 6-ring PAHs were $68.69 \pm 3.68 \%$ and $36.09 \pm 1.78 \%$, respectively. The average removal efficiency of the total PAHs after 80 days followed the order: R1M $(91.1 \%)>\mathrm{CKM}(84.9 \%)>\mathrm{CK}(77.6 \%)$, indicating that planted soil with inoculation of Mycobacterium sp. as well as non-planted soil inoculated with Mycobacterium sp. could both significantly accelerate the removal of PAHs compared to control soil. The bioavailability ratio of PAHs with 3 and 4 rings tended to decrease (from $59.9 \%$ to $14.8 \%$ for 3-ring and $7.61 \%$ to $5.08 \%$ for 4-ring, respectively in R1) while those with 5 rings increased significantly (from $2.41 \%$ to $33.78 \%$ in R1) during the last 40 days, indicating that bioavailability alteration varies with the number of rings in the PAHs. In addition, PAH bioavailability generally did not show a significant difference between treated soil and control soil. These results suggest that ryegrass rhizosphere effect as well as inoculation of Mycobacterium sp. can accelerate PAH removal in polluted soil. The bacteria community structure demonstrated a complex interplay of soil, bacteria and ryegrass root, and potential PAH degraders were present in abundance. This study provides the exploring data of rhizosphere and bioaugmentation effect on PAH dissipation in agricultural soil, as well as the change of bioavailability and microbial composition thereof.
\end{abstract}

Keywords Bioavailability $\cdot$ Community structure $\cdot$ PAHs $\cdot$ Rhizoremediation

\section{Introduction}

Polycyclic aromatic hydrocarbons (PAHs) pose local and international concerns owing to their strong carcinogenic, teratogenic and ecotoxic effects (Chen et al. 2016). The

Katarzyna Możdżeń

katarzyna.mozdzen@up.krakow.pl

Changxiong Zhu

zhuchangxiong@caas.cn

1 Institute of Environment and Sustainable Development in Agriculture, Chinese Academy of Agricultural Sciences, Beijing 100081, People's Republic of China

2 Institute of Biology, Pedagogical University of Krakow, Podchorążych 2 St, 30-084 Kraków, Poland hydrophobic property of PAHs keeps soil or sediment as the final sink of PAHs, making it rather important to achieve an effective remediation of polluted soil according to relevant cleanup standards (Peng et al. 2008). Rhizosphere is well known as a site for elevated microbial numbers and activities mainly through root development and exudation (Gentry et al. 2004). Thus rhizoremediation, as a method, can enhance PAHs degradation in polluted soils (Bourceret et al. 2015).

Several plants were reported in PAH rhizoremediation assays (Sipila et al. 2008; Pritchina et al. 2011; Bisht et al. 2014; Liu et al. 2014; Salehi et al. 2015; Shahsavari et al. 2015), among which ryegrass has been chosen as a research target due mainly to its large and fibrous root system that enables it to colonize a large area of soil and favors interactions between roots, microbes and pollutants (Joner et al. 2001; Corgi'e et al. 2006; Xu et al. 2014; Chen et al. 2016). The influence of 
plants on PAHs dissipation may vary according to the distance from growing roots (Bourceret et al. 2015), and it seems that PAH removal efficiency gets higher when proximity to roots increases (Corgi'e et al. 2003). However, rhizosphere zone has a clear definition which made collection of the rhizosphere soil quite complex in nature. Previous studies and experimental protocols have suggested vigorously shaking plant roots to separate bulk soil with rhizosphere soil (Barillot et al. 2013), which is a tedious process involving shaking for several minutes and solution preparation and yet not convenient to separate soil with the proximity to roots. In this present study, the three-chamber rhizoboxes were designed and used to compare the dissipation of PAHs in root area, near and far root area as well as bulk soil, which will be an easy and practical method to study rhizosphere. As for microbes, Mycobacterium spp. has been regarded as a promising genus associated with $\mathrm{PAH}$ degradation, especially pyrene (Uyttebroek et al. 2006).

Currently, the research for dissipation of PAHs has mainly focused on industrial soils due to their severity of contamination based on concentration, and only a few studies have explored contaminated agricultural soils (Duan et al. 2015; Guo et al. 2016; Peng et al. 2016). However, agricultural contaminated soils may pose a more direct risk since they provide food for humans and farmers expose to PAHs during tilling. Previous investigation of PAHs in Shanxi province, China found that the total 16 priority PAHs ranged from 294 to $1665 \mathrm{ng} / \mathrm{g}$ in agricultural soil, indicating a heavy pollution existed in agricultural soil (Duan et al. 2015). The primary source of PAHs in agricultural soils, may be wastewater irrigation, open burning of straw and brushwood, coal and wood usage for cooking or heating, and atmospheric deposition (Maliszewska-Kordybach 1996; Peng et al. 2016), resulting in different bio-availabilities to those in industrial contaminated soils. In agricultural lands, bioaccumulation of PAHs, as an exposure pathway, is distinct from that in industrial lands, and is of high risk in spite of the lower concentration in soils (Srogi 2007). Therefore, soil remediation and bioavailability assessment for PAHs in contaminated agricultural soils are especially important issues. In this study, bioavailability assessment was done by using Tenax-TA extraction method and was compared between soils collected from different compartments of rhizoboxes. The two in combination, as far as we know, has not been done by previous studies.

The main object of this study is thus to investigate the rhizosphere and bioaugmentation effect on PAH dissipation in agricultural soil, the change of bioavailability of PAHs, and the structure of microbial community after planting ryegrass and inoculating a Mycobacterium sp. strain. All the soil for above experiments were collected from comparted rhizoboxes. This study helps to provide the bioremediation and rhizosphere effect from multiple perspectives.

\section{Materials and methods}

\section{Soil, plant and microorganism}

The agricultural soil used in this study was sampled from the top $20 \mathrm{~cm}$ of a waste water irrigated site with a long history of contamination in a sub-urban area in Shenyang, China. The physiochemical properties of the soil were: $\mathrm{pH} 8.25$, total nitrogen $0.62 \mathrm{~g} / \mathrm{kg}$, soil organic carbon $1.57 \%$, and total phosphorus $1.37 \mathrm{~g} / \mathrm{kg}$. The soil was sieved through a $2 \mathrm{~mm}$ mesh, homogenized, and kept in $4{ }^{\circ} \mathrm{C}$ until analysis.

The rhizobox used was a compartmentalized device made of polymethyl methacrylate. The size of the rhizobox was $20 \mathrm{~cm}$ long $\times 15 \mathrm{~cm}$ wide $\times 20 \mathrm{~cm}$ high. Each rhizobox was divided into five compartments with three sections: the central compartment, or the root area, left and right near root area, and left and right far root area, respectively (Fig. 1). Ryegrass seeds (Lolium perenne L.) were so densely sown that by the time we began sampling, the root area soil was full of ryegrass roots and could be taken as rhizosphere (less than $2 \mathrm{~mm}$ from the root surface). When sampling, a small stainless-steel spoon was used to take out soil with care around the roots of ryegrass, made sure the ryegrass would not be eliminated. However, if any were eliminated inadvertently, we would sow new ryegrass seeds immediately. Each compartment was $3.5 \mathrm{~cm}$ wide with a removable front face made of nylon mesh $(<25 \mu \mathrm{m})$ and holes of $0.4 \mathrm{~cm}$ diameter to avoid water stagnation at the bottom. Each rhizobox was filled with contaminated soil. The moisture of soil was measured by drying it at $105^{\circ} \mathrm{C}$ for six hours in oven and using an electronic balance to measure the change of weight. Ryegrass seeds were planted in the root area, either inoculated or uninoculated with Mycobacterium sp. with three replicates. Before germination, the rhizoboxes were kept in a dark condition. However, after germination, the rhizoboxes were placed with controlled conditions in a plant growth chamber $\left(25{ }^{\circ} \mathrm{C} / 18{ }^{\circ} \mathrm{C}\right.$ day/night, $60 \%$ relative humidity) and were watered to maintain soil humidity at $60 \%$ of the soil water holding capacity by watering to weight weekly with distilled water.

The microbial strain used in this study was with a $100 \%$ homogeneity to Mycobacterium gilvum (GenBank accession number DQ512892), and was isolated previously from a PAH heavily polluted soil. The strain was maintained at $4{ }^{\circ} \mathrm{C}$ and periodically sub-cultured on Luria-Bertani (LB) agar plates. Before the inoculation in this study, pre-inoculum of Mycobacterium sp. was grown in liquid LB medium for 7 days at $28^{\circ} \mathrm{C}$ in $250-\mathrm{mL}$ Erlenmeyer flasks and then transferred to 2-L Erlenmeyer flasks for a $10 \%(v / w)$ inoculum into the soil for inoculation treatments. After a thorough mix of liquid 
Fig. 1 Structure of the designed rhizobox

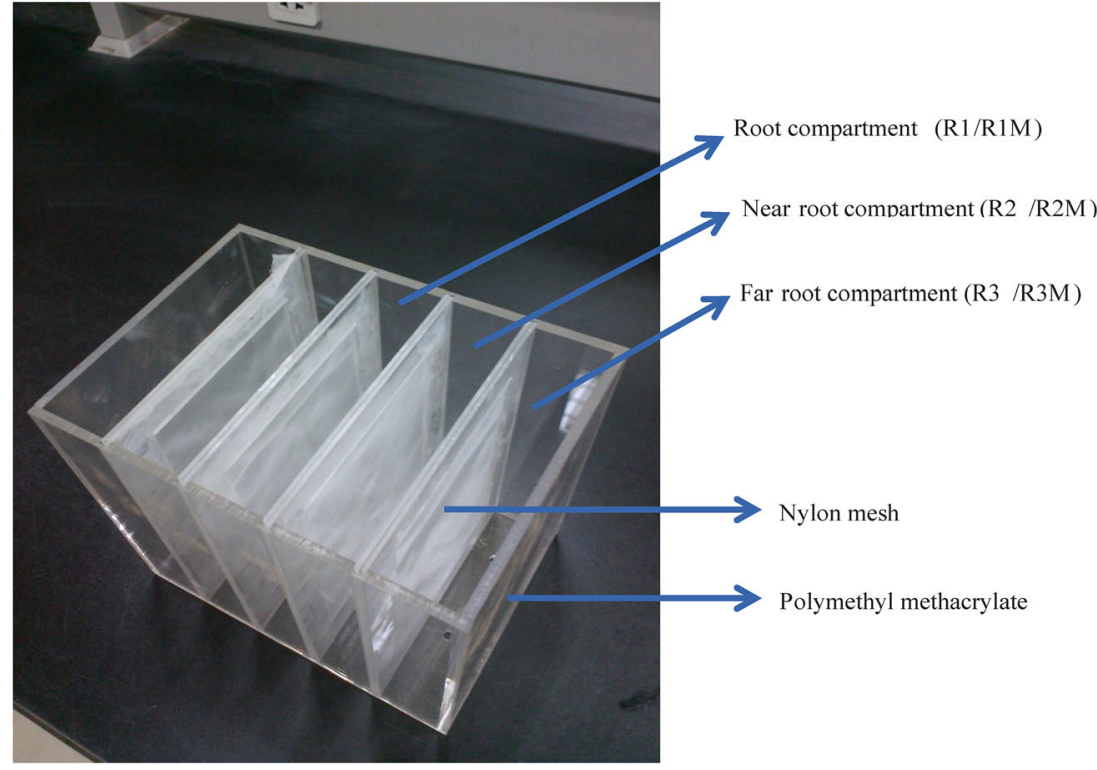

medium containing Mycobacterium sp. into soil, the soil was put in the rhizoboxes for sowing and non-sowing with ryegrass seeds. For treatments without inoculation of Mycobacterium sp., the same volume of sterile LB medium was thoroughly mixed with the soil.

Eight treatments were carried out for analysis of PAHs dissipation and bioavailability including: the root area (R1), near root area (R2), far root area (R3), unplanted soil (CK), root area with Mycobacterium sp. (R1M), near root area with Mycobacterium sp. (R2M), far root area Mycobacterium sp. (R3M), and unplanted soil with Mycobacterium sp. (CKM). Ryegrass seeds were planted in the root area. Soil samples were collected after 20 days, 40 days and 80 days from different areas of the root in triplicate for analysis.

\section{PAH extraction from soil}

PAHs in the soil were extracted using a mechanical chemical extraction procedure (Wu et al. 2010; Guo et al. 2016). $5 \mathrm{~g}$ soil was put into a glass bottle, then $1.5 \mathrm{~g}$ of sodium chloride, $10 \mathrm{~mL}$ of distilled water, $15 \mathrm{~mL}$ of dichloromethane, and $20 \mathrm{~mL}$ of acetone were added. All bottles were tightly capped and shaken at $160 \mathrm{rpm}$ on an orbital shaker for $16 \mathrm{~h}$. The upper layer organic phase was concentrated and cleaned up by passing it through a chromatography column filled with $1 \mathrm{~g}$ of activated silica gel (heated at $130{ }^{\circ} \mathrm{C}$ for $16 \mathrm{~h}$ ) and $0.5 \mathrm{~g}$ of deactivated aluminum oxide (deactivated by addition of $15 \%$ water). The collected sample was then evaporated to dryness under nitrogen gas, and finally was re-dissolved in acetonitrile for analysis by highperformance liquid chromatography (HPLC) (Dionex Ultimate 2000, USA).

\section{PAH extraction via Tenax-TA}

Tenax-TA (177-250 $\mu \mathrm{m}, 60-80$ mesh; Alltech Associates, Inc., Chicago, Illinois, USA.) is a porous polymer based on 2,6-diphenyl-p-phenylene oxide. Before the experiment, the Tenax-TA was ultrasonically cleaned first with water, followed by hexane and acetone (three times each; $10 \mathrm{~mL}$ solvent $\mathrm{g}^{-1}$ Tenax-TA) and dried at $110{ }^{\circ} \mathrm{C}$ overnight. The Tenax-TA extraction experiment was carried out using the method described by Guo et al. (2016). On the fourth day (the corresponding time of fast extraction in this study), the tubes were taken out, centrifuged at $1609 \times \mathrm{g}$ for $20 \mathrm{~min}$ to ensure that the Tenax-TA beads all floated to the surface. The Tenax-TA beads were then collected using a stainless-steel scoop and ultrasonically extracted three times for $30 \mathrm{~min}$ each time with $20 \mathrm{~mL}$ of a hexane-acetone mixture $(3: 1 \mathrm{v} / \mathrm{v})$. The three extracts were combined for each sample and concentrated via rotary evaporation, dried under a nitrogen gas stream, dissolved in $2 \mathrm{~mL}$ acetonitrile, and measured using HPLC.

\section{Analysis of microbial consortia}

For the microbial community analysis, the total genomic DNA in soil was extracted using Mo bio PowerSoil DNA Isolation Kit. For DGGE (denaturing gradient gel electrophoresis) analysis, primers GC clamp-341F (5'-CGCC CGCCGCGCGCGGCGGGCGGGGCGGGGGCACGGGG GGCCTACGGGAGGCAGCAG-3') and 534R (5'-ATTA CCGCGGCTGCTGG-3') were used to amplify the variable V3 region of bacteria 16S rDNA genes. DNA was amplified in a Professional Thermocycler (Biometra, Germany) using the following amplification program: $95^{\circ} \mathrm{C}$ for $5 \mathrm{~min}(1 \mathrm{cy}-$ cle), $95^{\circ} \mathrm{C}$ for $30 \mathrm{~s}, 56^{\circ} \mathrm{C}$ for $30 \mathrm{~s}, 72^{\circ} \mathrm{C}$ for $30 \mathrm{~s}(35$ cycles $)$, $72{ }^{\circ} \mathrm{C}$ for 5 min $(1$ cycle). PCR products were screened by 
electrophoresis in $1 \%(\mathrm{wt} / \mathrm{vol})$ agarose gel to verify the amplification and the size of amplified DNA (194 bp expected). The DGGE denaturing gel was prepared with a continuous gradient of denaturants (from $40 \%$ to $60 \%$ ) in $10 \%$ polyacrylamide gel. The PCR products were separated with a DCode electrophoresis system (BioRad Laboratories, USA) operated at a constant voltage of $45 \mathrm{~V}$ for $16 \mathrm{~h}$ at $60{ }^{\circ} \mathrm{C}$. To determine the sequence of the separated fragments, individual DGGE bands of DNA bands were excised and suspended in $30 \mu \mathrm{L}$ of distilled water overnight.

For cloning and sequencing with the diffused PCR-DGGE products as template, DNA fragments were re-amplified using $341 \mathrm{~F}$ (without GC clamp) and 534R primers, then the DNA amplicons were visualized by electrophoresis in $1 \%(\mathrm{w} / \mathrm{v})$ agarose gel, excised, gel purified, ligated into PMD19-T vectors (pMD ${ }^{\mathrm{TM}} 19-\mathrm{T}$ Vector Cloning Kit), and transformed into competent cells (Escherichia coli DH5 $\alpha$, Takara Bio Inc.). Bacteria were grown in LB medium in which IPDG, X-Gal and Ampicillin were added. Five white colonies per sample were randomly picked and sequenced. All sequences obtained were submitted to GenBank at National Center for Biotechnology Information (NCBI).

\section{Data analysis}

The experimental results such as the data of removal ratio and bioavailability of PAHs, were evaluated and least significance difference using SPSS 16.0 statistical software at 95\% confidence by Student t-tests $(p<0.05)$, one-way ANOVA followed by Duncan multiple comparison test and letters (a, b, c) indicate significant differences $(p<0.05)$. SigmaPlot 10.0 was used to generate the graphs.

\section{Results and discussion}

\section{The concentrations of PAHs in the original soil and removal efficiency during the study}

The PAH concentration in the original soil were listed in Table 1. The 3- (37.1\%) and 4-ring PAHs (42.1\%) dominated in the soil, with phenanthrene, fluoranthene and pyrene having the highest concentration. The total concentration of 12 PAHs was $10.85 \mathrm{mg} / \mathrm{kg}$. Previously, a contamination classification method was established by Maliszewska-Kordybach (1996) and has been widely used nowadays, in which the contamination level of soil was classified into four categories, namely non-contaminated $(<200 \mathrm{ng} / \mathrm{g})$, weakly contaminated $(200$ $600 \mathrm{ng} / \mathrm{g})$, contaminated (600-1000 ng/g) and heavily contaminated soils (> $1000 \mathrm{ng} / \mathrm{g}$ ). According to this standard, our soil samples were heavily contaminated. Soil was sampled to test the rhizosphere effect 20 days after ryegrass sowing. All of the ryegrass seeds germinated in contaminated soil within 7 days and on day 20, their roots were dense enough to make the surrounding soil be taken as rhizosphere soil. Analysis of PAH removal efficiency showed the active rhizosphere effect of ryegrass on the dissipation of these pollutants from soil (Fig. 2).

In treatments without Mycobacterium sp. inoculation, the removal efficiency of PAHs was significantly higher in planted soil compared to unplanted soil (CK) for after 80 days (Fig. 2). At the early stage (20 days), the removal efficiency in R1 (root area) and R2 (near root area) was mostly higher than that in R3 (far root area) and CK, but with increasing treatment periods, the removal efficiency in R3 narrowed the gap with R1 and R2, and even exceeded the later. This might be ascribed to the synergy between plants and the indigenous
Table 1 Concentrations of PAHs in the original polluted soil

\begin{tabular}{llll}
\hline Compounds & Rings & $\begin{array}{l}\text { Concentrations } \\
{\left[\mathrm{mg} \mathrm{kg}^{-1}\right]}\end{array}$ & Tenax-TA extractions $\left[\mathrm{mg} \mathrm{kg}^{-1}\right]$ \\
\hline Phenanthrene & 3 & $3.45 \pm 0.24$ & $0.51 \pm 0.04$ \\
Anthracene & 3 & $0.58 \pm 0.03$ & $0.02 \pm 0.01$ \\
Fluoranthene & 4 & $2.02 \pm 0.13$ & $0.07 \pm 0.03$ \\
Pyrene & 4 & $1.40 \pm 0.07$ & $0.14 \pm 0.01$ \\
Benzo(a)anthracene & 4 & $0.64 \pm 0.04$ & $0.018 \pm 0.01$ \\
Chrysene & 4 & $0.51 \pm 0.09$ & $0.015 \pm 0.01$ \\
Benzo(b)fluoranthene & 5 & $0.61 \pm 0.08$ & $0.004 \pm 0.01$ \\
Benzo(k)fluoranthene & 5 & $0.25 \pm 0.03$ & $0.003 \pm 0.01$ \\
Benzo(a)pyrene & 5 & $0.35 \pm 0.10$ & $0.007 \pm 0.01$ \\
Dibenz(ah)anthracene & 5 & $0.09 \pm 0.03$ & $\mathrm{ND}$ \\
Benzo(ghi)perylene & 6 & $0.58 \pm 0.12$ & $\mathrm{ND}$ \\
Indeno(cd)pyrene & 6 & $0.36 \pm 0.09$ & $\mathrm{ND}$ \\
Total PAHs & & $10.85 \pm 0.89$ & $0.79 \pm 0.02$ \\
\hline
\end{tabular}

* Note: ND indicates not detected 
Fig. 2 The removal percentage of PAHs with different rings. R1: root area; R2: near root area; R3: far root area; CK: unplanted control soil; R1M: root area + Mycobacterium sp.; R2M: near root area + Mycobacterium sp.; R3M: far root area + Mycobacterium sp.; CKM: unplanted soil + Mycobacterium sp. The lower-case letters represent significant difference between R1, R2, R3 and CK, or between R1M, R2M, R3M and CKM $(p<0.05)$; capital letters indicate a significant difference between the inoculated treatment and the corresponding noninoculated treatment $(p<0.05)$
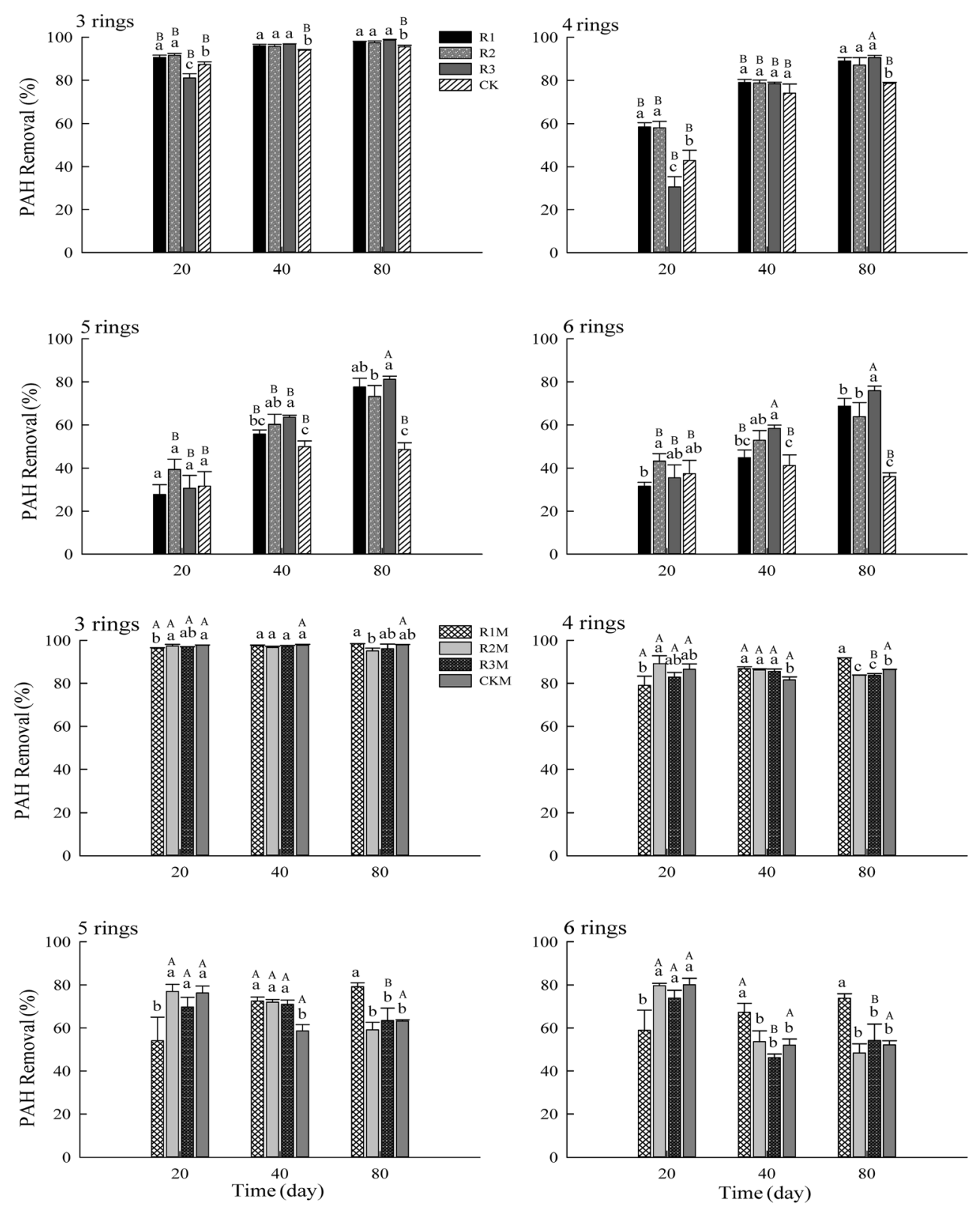

microorganisms in PAH dissipation. Although the initial synergy between plants and microorganisms were in the rhizosphere (R1), the root exudates and the corresponding adapted microorganism could penetrate the nylon mesh into R2 and R3 through frequently watering. As a result, ryegrass growth accelerated PAH dissipation in all R1-R3 areas compared to the non-planted treatment $(\mathrm{CK})$, and this effect continue to help with PAH dissipation over time in 80 days. The removal improvement by planting ryegrass existed among all PAHs with 3-6 rings, especially on 4-6 rings PAHs, as a bigger disparity could be seen between root area (R1) and bulk soil (CK) for these HMW PAHs. For example, in the root area (R1) and bulk soil (CK), the removal of 3-ring PAHs were $97.72 \pm 0.34 \%$ and $95.51 \pm 0.75 \%$, 4-ring PAHs were $89.01 \pm$ $1.61 \%$ and $78.65 \pm 0.47 \%$, 5-ring PAHs were $77.64 \pm 4.05 \%$ and $48.63 \pm 3.19 \%$, 6-ring PAHs were $68.69 \pm 3.68 \%$ and $36.09 \pm 1.78 \%$, respectively (Fig. 2). More importantly however, HMW PAHs have been reported to be more carcinogenic and mutagenic than LMW PAHs (Kanaly and Harayama 2010; Aziz et al. 2014), which highlights the advantage of rhizoremiation by ryegrass and bioaugmentation by Mycobacterium sp. in this study. The similarity of removal efficiency on 3-ring PAHs between treatments was also because they were usually more easily to degrade.

Rhizosphere effect in the treatments inoculated with Mycobacterium sp. was also conspicuous but had different pattern with those non-inoculated treatments. At the early stage (before 20 days), the dissipation of 3- and 6-ring PAHs was significantly higher in Mycobacterium sp. inoculated soil than the corresponding non-inoculated area of soil $(p<0.05)$, 
and the same trend went on till 40 days for 4- and 5-ring PAHs (Fig. 2). However, it is worth noting that the removal of 4- to 6-ring PAHs in R1M was significantly lower than R2M, R3M and CKM before 20 days $(p<0.05)$. As time went on, the removal efficiency of PAHs in R1M eventually exceeded those in the other 3 treatment groups. This indicates that the expected positive interaction of ryegrass roots with inoculated Mycobacterium sp. in PAH dissipation may need some time to manifest. While inoculation of Mycobacterium sp. did show a significant acceleration of PAH dissipation in CKM soil than CK soil $(p<0.05)$ the interaction between ryegrass roots and the inoculants seemed somewhat to have retarded this bioaugmentation effect at an early stage. This could be ascribed to some complicated mechanisms that are currently unknown, and more studies are needed to address this question. Nevertheless, R1M still showed significant acceleration in 3and 4-ring PAH dissipation than R1 in the early 20 days. The mechanisms of different proximity to plant roots could obtain different PAH removal efficiency mainly reside in the root exudates that interact with the microorganisms, which is also the root cause of rhizosphere effect (Corgi'e et al. 2003). Moreover, basically the dissipation of PAHs was faster in Mycobacterium sp. inoculated soil, which could be seen if the CKM and CK were compared (Fig. 2). This indicates that the strain used in this study was indeed with higher efficiency in PAH dissipation. Although the data for 5- and 6-ring PAHs were abnormal to some extent since the removal efficiency declined in groups of R2M, R3M and CKM with increasing time due to unknown reasons, our results still indicates the rhizoremediation of ryegrass and bioaugmentation of Mycobacterium sp. alone and combined are promising methods in accelerating PAH dissipation.

In the present study, both the bioaugmentation and the planted treatments could enhance the dissipation of PAHs, especially the removal of HMW PAHs, which was consistent with other reports (Xu et al. 2014; Hou et al. 2015). In our present study, the average removal efficiency of the total PAHs after 80 days followed the order: R1M (91.1\%)> CKM (84.9\%) > CK (77.6\%), indicating that planted soil with inoculation of Mycobacterium sp. as well as non-planted soil inoculated with Mycobacterium sp. could both significantly accelerate the removal of PAHs compared to control soil. In addition, the dissipated percentages of total PAHs in this study were comparable with the study of Tejeda-Agredano et al. (2013), which reported the rhizosphere effect of sunflower and obtained total removal efficiency of 6 PAHs (all with 3 to 4 rings) $93.1 \%$ in planted soil and $77.1 \%$ in unplanted soil during 90 days study. Wang et al. (2013), also found that the removal of phenanthrene and pyrene could reach above $91 \%$ in the Sedum alfredii and ryegrass co-planted soil within 90 days (Wang et al. 2013). However, the removal efficiency of total PAHs in this study was much higher than that reported by $\mathrm{Xu}$ et al. (2014) and Hou et al. (2015) (at most 62.6\% and
$69.6 \%$, respectively, in microbial/phytoremediation treatment), whose total PAHs concentration was more than 10 times as much as the present report; and even higher than the values reported by $\mathrm{Fu}$ et al. (2012), in which the initial total concentration of PAHs in ryegrass planted soil was only $13 \%$ of the present study. Therefore, it is assumed that the initial pollution level of PAHs played an important role in the dissipation quantity of PAHs. In fact, PAH contamination level may play a decisive role in bacterial community structure by affecting the distribution, diversity, and levels of rhizosphere bacteria (Peng et al. 2015), and this might also substantially affect the removal efficiency of PAHs. Other factors influencing the removal efficiency of PAHs might be the microbial community structure, soil environmental condition, plant species and the profile of PAHs.

\section{The bioavailability assessment of PAHs via Tenax-TA extraction}

The Tenax-TA extraction in this study failed to extract 6-ring PAHs due to their extremely low concentration and bioavailability. As for 3- to 5-ring PAHs, the initial Tenax-TA extraction concentrations for individual PAHs were listed in Fig. 3. However, after 20 days, only three PAHs (anthracene, chrysene, benzo(b)fluoranthene) were successfully extracted and suitable for comparison, other individual PAHs were similar to 6-ring PAHs, often did not show their concentrations after determination by using HPLC. Therefore, only these three PAHs were analyzed in this section as the representatives for 3-, 4- and 5-ring PAHs, respectively. As it is shown in Fig. 3, the extraction of anthracene and chrysene decreased significantly from day 40 to day 80 , while benzo(b)fluoranthene increased significantly at the same period, regardless of whether Mycobacterium sp. was inoculated or not. If we define the Tenax-TA extracted PAH concentration divided by the total remaining concentration of corresponding $\mathrm{PAH}$ as the bioavailability ratio, the bioavailability ratio of PAHs with 3 and 4 rings tended to decrease (from $59.9 \%$ to $14.8 \%$ for $3-$ ring and $7.61 \%$ to $5.08 \%$ for 4-ring, respectively) in R1 while those with 5 rings increased significantly (from $2.41 \%$ to $33.78 \%$ ) in $\mathrm{R} 1$ from 40 to 80 days during the last 40 days, indicating that bioavailability alteration varies with the number of rings in the PAHs. The decrease in Tenax-TA extracted anthracene and chrysene might be due to the continuing reduction of their residual in the soil, while the sharp increase of benzo(b)fluoranthene after 40 days could be ascribed to the promotion of desorption from soil, which might be caused by the biosurfactants produced by microorganism (Tyagi et al. 2011) and root exudates (He and Chi 2015). The rationality of this assumption could be further improved by the percentage of Tenax-TA extraction in the mechanical chemical extraction, which showed this percentage of benzo(b)fluoranthene was less in CK than in planted or inoculated soil (Fig. 3). In the 

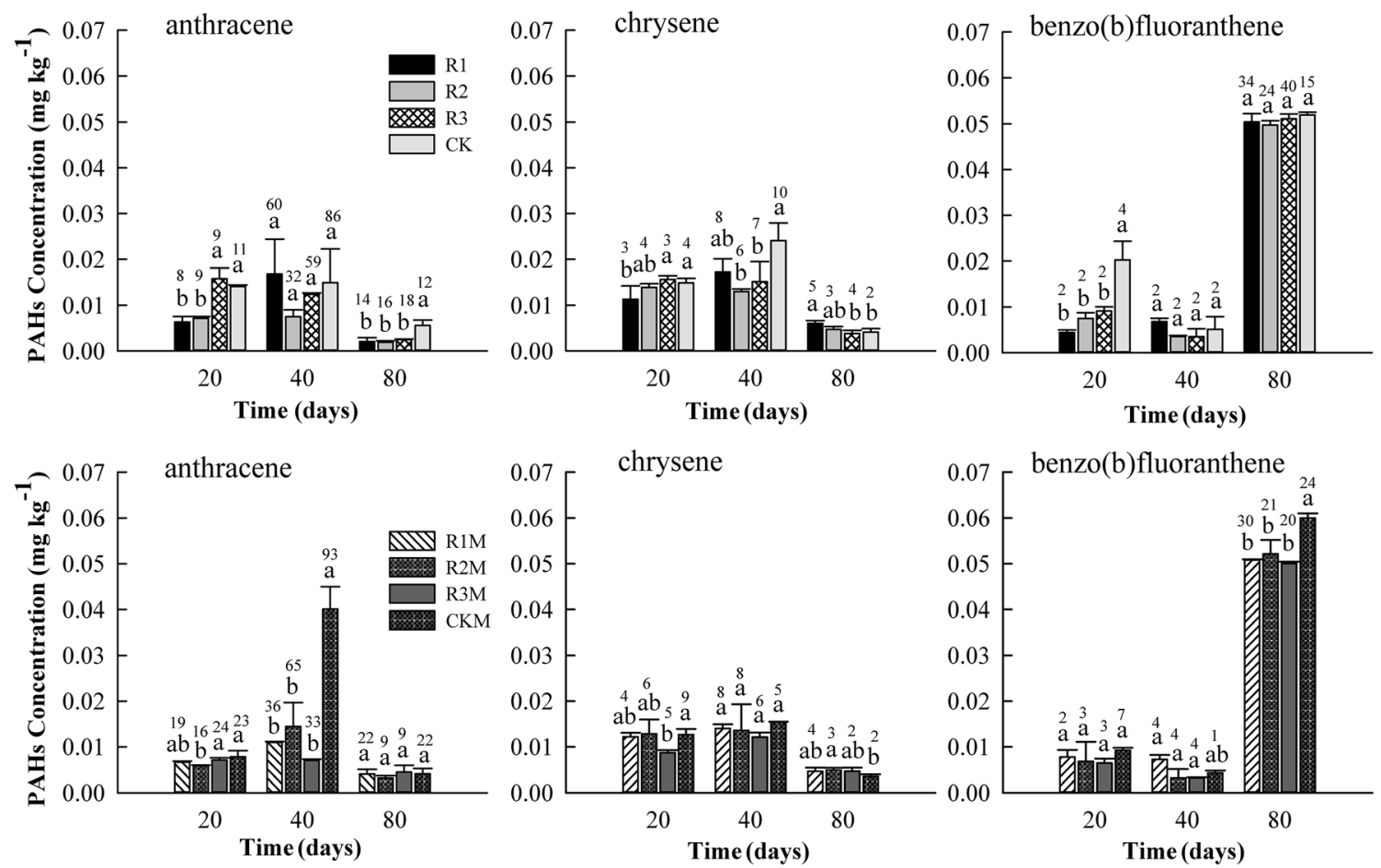

Fig. 3 Tenax-TA extraction of 3 representative PAHs. The numbers above the columns are the percentage (\%) of Tenax-TA extraction to the corresponding mechanical chemical extraction. Lower-case letters are the result of multiple comparison between treatments (ANOVA, $p<0.05$ )

actual sense, this phenomenon of increase in desorption of benzo(b)fluoranthene overtime should be considered during remediation studies because the increase in $\mathrm{PAH}$ desorption can enhance the further dissipation of these PAHs. On the other hand, however, the increase in desorption may pose deleterious effects on the ecological environment and human health, especially the desorption of 5-6 ring PAHs which are more toxic.

Furthermore, in the present study, there was no significant difference in quantity of Tenax-TA extraction between Mycobacterium sp. inoculated and non-inoculated treatments. This is probably because that the disparity of PAHs level was not big enough to generate a significant difference in TenaxTA extraction. Meanwhile, the similarity in amount of TenaxTA extraction between treatments indicates that the bioavailability of PAHs was similar in most cases in the present study. This means the current phytoremediation and bioaugmentation may not necessarily significantly modify the bioavailability of PAHs although both of them can accelerate the dissipation of PAHs.

\section{DGGE patterns of the bacterial communities}

The DGGE profile of all the samples collected at three different incubation phases were shown in Fig. 4. The banding patterns between all the samples were different from each other. Forty-eight of detectable DGGE bands were excised and sequenced, and the NCBI database and nucleotide
BLAST (http://blast.ncbi.nlm.nih.gov/Blast.cgi) was used. As a result, 42 sequences in total were obtained (Table 2). Variations in bacterial communities were observed over the entire study periods. The DGGE fingerprints obtained on day 20 showed relatively more similar banding profiles than those on day 40 and 80 , indicating that homogeneity of microbial communities under different conditions existed at an early stage. This showed that there were likely more dominant species were present on day 20 in view of the band intensity. Moreover, some bands existed throughout the period while other bands were only present at a certain stage. For instance, band 20.11, 40.11 and 80.10 shared the same sequence and dominated from day 20 to day 80 . DGGE bands pattern also varies between treatments, for example, band 40.08 and 80.06 existed in all the soil samples amended with Mycobacterium sp. but were not detected in those treatment without Mycobacterium sp. amendment; band 40.10 were detected in most treatments except R1 and R1M; band 80.07 was only discernible in R1, R2, R1M and R2M, indicating that both the presence of plant and soil areas could determine the nature of bacterial community. It has been reported that microbial communities can be considerably different from rhizosphere and soil area without a direct influence of roots (Tejeda-Agredano et al. 2013), which could also be seen in the present study.

Parrish et al. (2005) used tall fescue and yellow sweet clover to study rhizosphere effect and found that there was a shift in the microbial community over time. These phenomena 
Table 2 Sequencing result of DGGE bands

\begin{tabular}{|c|c|c|c|}
\hline Band & Phylogenetic group & Closest sequence (Accession NO.) & Homology $[\%]$ \\
\hline 20.06 & \multirow[t]{9}{*}{ Actinobacteridae } & Blastococcus aggregatus (AB685271.1) & 100 \\
\hline 20.07/80.07 & & Streptomyces longisporoflavus strain VSA18 (KT895215.1) & 100 \\
\hline 20.08 & & Arthrobacter oxydans strain 8-2 (KU362288.1) & 100 \\
\hline 20.14 & & Micromonospora chokoriensis strain FoRh32 (KM370057.1) & 99 \\
\hline 40.15 & & Methyloceanibacter caenitepidi DNA (AP014648.1) & 99 \\
\hline 40.16 & & Aquihabitans daechungensis strain CH22-21 (NR_132289.1) & 100 \\
\hline 40.17 & & Arthrobacter oxydans strain 8-2 (KU362288.1) & 100 \\
\hline 80.14 & & Nocardioides furvisabuli strain M0613 (KF924228.1) & 100 \\
\hline 80.16 & & Mycobacterium houstonense strain ATCC 49403(LT223693.1) & 99 \\
\hline 20.03 & \multirow[t]{13}{*}{ Alphaproteobacteria } & Methylobacteriaceae bacterium UAPS0137 (KT936113.1) & 99 \\
\hline 20.04 & & Sphingomonas gimensis strain 9PNM-6 (NR_134181.1) & 100 \\
\hline 20.12 & & Sphingomonas kyeonggiensis strain THG-DT81 (NR_134182.1) & 99 \\
\hline 20.13/80.15 & & Sphingomonas gei strain ZFGT-11 (NR_134812.1) & 100 \\
\hline 40.02 & & Erythrobacteraceae bacterium LSUCC0267 (KU382434.1) & 100 \\
\hline 40.14 & & Sphingobium baderi strain DE-13 (CP013264.1) & 100 \\
\hline 80.01 & & Pseudochelatococcus contaminans strain MPA 1105 (KJ886940.1) & 98 \\
\hline 80.08 & & Sphingobium baderi strain DE-13 (CP013264.1) & 100 \\
\hline 20.10 & & Psychrobacillus psychrodurans strain NB-9 (KU254665.1) & 100 \\
\hline $20.11 / 40.11 / 80.10$ & & Bacillus thuringiensis strain 3-1 (KU362281.1) & 100 \\
\hline $40.03 / 80.11$ & & Bacillus megaterium gene (LC092937.1) & 100 \\
\hline 40.12 & & Bacillus firmus strain BS9 (KU052622.1) & 100 \\
\hline 40.13 & & Paenibacillus terrigena strain LEM33 (KU180355.1) & 97 \\
\hline 20.01 & \multirow[t]{6}{*}{ Gammaproteobacteria } & Pseudomonas fluorescens strain HRA32 (KU132384.1) & 100 \\
\hline 20.09 & & Pseudomonas fluorescens strain F-38 (KU305728.1) & 100 \\
\hline 40.06 & & Pseudomonas frederiksbergensis strain NSMA50 (KT321279.1) & 99 \\
\hline 80.05 & & Salinimonas lutimaris strain DPSR-4 (NR_109101.1) & 93 \\
\hline 40.08 & & Pseudomonas aeruginosa strain Dr10R (JF513141.1) & 99 \\
\hline 80.04 & & Lysobacter antibioticus strain JP28 (KU613064.1) & 100 \\
\hline 40.05 & \multirow[t]{4}{*}{ Flavobacteriia } & Flavobacterium psychrophilum strain Z2 (CP012586.1) & 95 \\
\hline 40.07 & & Flavobacterium piscis strain 412R-09 (NR_133746.1) & 100 \\
\hline $40.10 / 80.09$ & & Salinimicrobium gaetbulicola strain BB-My20 (NR_109368.1) & 99 \\
\hline 80.13 & & Flavobacteriaceae bacterium MAR_2010_199 (JX854343.1) & 96 \\
\hline 20.02 & \multirow[t]{2}{*}{ Betaproteobacteria } & Achromobacter ruhlandii strain DRK12 (KT231777.1) & 100 \\
\hline 20.05 & & Shinella kummerowiae strain GYB33 (JQ342936.1) & 100 \\
\hline 40.09 & \multirow[t]{2}{*}{ Deltaproteobacteria } & Bdellovibrio bacteriovorus strain $109 \mathrm{~J}$ (CP007656.1) & 92 \\
\hline 80.03 & & Desulfovibrio alaskensis G20 strain G20 (NR_074749.1) & 89 \\
\hline 40.01 & \multirow[t]{2}{*}{ Sphingobacteriia } & Niabella terrae strain ICM 1-15 (NR_132698.1) & 98 \\
\hline 40.04 & & Olivibacter soli strain E6 (KT634064.1) & 99 \\
\hline 80.02 & Bacteroidetes & Uncultured Bacteroidetes bacterium HG-B0273 (JN409173.1) & 99 \\
\hline 80.12 & Chloroflexia & Agricultural soil bacterium CRS5639T18-1 (HQ132683.1) & 95 \\
\hline 80.06 & Clostridia & Clostridium ultunense strain DSM 10521 (NR_117379.1) & 99 \\
\hline 80.17 & Nitrospira & Candidatus Saccharibacteria oral taxon TM7x (CP007496.1) & 96 \\
\hline
\end{tabular}

Note: The number before points are the sample time (day); and after points are the corresponding number labeled in DGGE

might be caused by the root exudates in the soil which have induced chemotaxis and relevant mobilization mechanisms for motile microorganisms (TejedaAgredano et al. 2013). Previous reports concluded that changes of the root exudate composition caused by plant species and the developmental stage of plants could obviously affect the rhizospheric community (Bisht et al. 2015; Bourceret et al. 2015). 
Fig. 4 DGGE profiles. a) day 20; b) day 40 ; c) day 80 . The lanes $1-$ 7 are treatments R1, R2, R3, R1M, R2M, R3M, CK, respectively. Bands attached with numbers and arrows were excised for further analysis. The left unlabeled lane is Marker DL2000 as a reference of band position

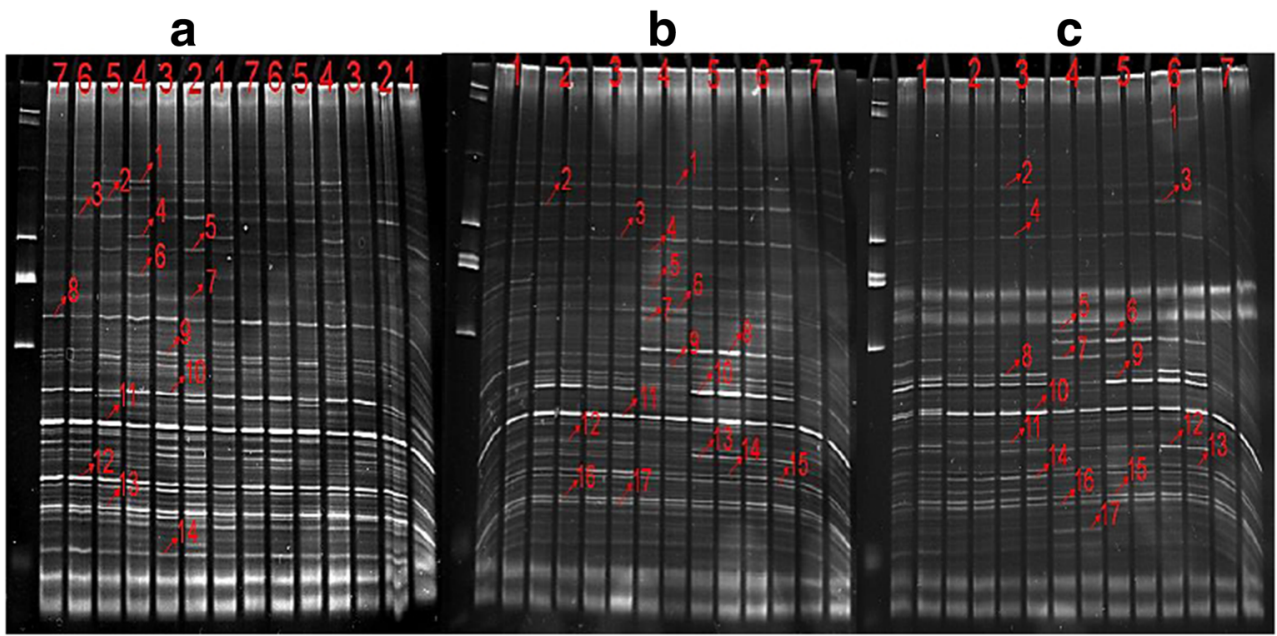

\section{Taxonomic composition analysis}

Taxonomic analysis showed that the total 42 bacterial sequences could be classified into six phyla (Actinobacteria, Proteobacteria, Chlorofexi, Bacteroidetes, Firmicutes, Nitrospira) and twelve classes (Actinobacteridae, Alphaproteobacteria, Betaproteobacteria, Deltaproteobacteria, Gammaproteobacteria, Chloroflexia, Flavobacteriia, Sphingobacteriia, Bacilli, Bacteroidetes, Clostridia and Nitrospira), the relative abundance of the phylogenetic groups based on classes are shown in Fig. 5. Proteobacteria and Actinobacteria were two dominant phyla in the studied soil. At the class level, Actinobacteridae accounted for $21.43 \%$, followed by Alphaproteobacteria (19.04\%), Gammaproteobacteria (14.29\%), Bacilli (11.90\%) and so on. To date, Proteobacteria is the phylum most identified as PAH degraders among all the species reported as hydrocarbondegrading bacteria, and representatives of the Actinobacteria, Firmicutes and Bacteroidetes phyla also have been frequently encountered (Røberg et al. 2011). Therefore, these dominant phyla might have played a leading role in the dissipation of
PAHs in this study. Some genera of these phyla have already been reported to be able to degrade PAHs. For example, many Mycobacterium strains, which belongs to Actinobacteria, contain nidA genes and could degrade high molecular weight (HMW) PAHs (DeBruyn and Sayler 2009), their capability of degrading PAHs often exceed that of Sphingomonas strains (belonging to Alphaproteobacteria) (Uyttebroek et al. 2006) which can also degrade phenanthrene, fluoranthene, pyrene and so on.

According to the DGGE profiles (Fig. 4) and their sequencing and aligning result (Table 2), it is clear that the bands $20.11,40.11$ and 80.10 were dominant throughout the experimental period and shared the same sequence, with an $100 \%$ similarity to a Bacillus thuringiensis strain. B. thuringiensis has been reported to be able to degrade HMW PAHs like fluoranthene and pyrene (Maiti et al. 2012). Also, the genus of Bacillus is one of typical genera that can metabolize PAHs (Ferradji et al. 2014), some of which can even utilize benzo(a)pyrene as sole carbon source (Lily et al. 2010). In addition, it is reported that bacteria which have survived the adverse effects of PAHs in contaminated soil sites are likely to
Fig. 5 Histogram of the relative abundance of the major bacteria phylogenetic groups

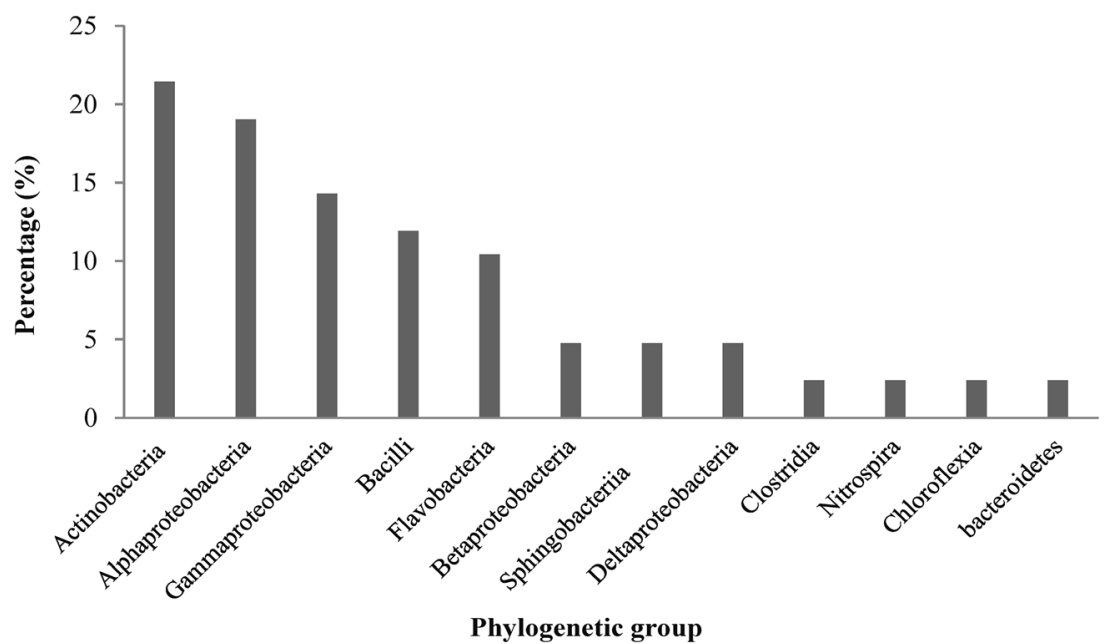


play a leading role in PAH biodegradation (Khanna et al. 2011). Therefore, the prevalence of the strain represented by the bands $20.11,40.11$ and 80.10 might be significantly important in PAH degradation in present study. In addition, we failed to analyze the dynamics of the Mycobacterium sp. strain in the present study, which might because the bands of Mycobacterium sp. were missed on the DGGE gel while some other bands were excised as not all the discernable bands were excised (Fig. 4), or simply because Mycobacterium sp. did not show a discernable band due to low abundance. Nevertheless, our present study was able to evaluate the bioaugmentation effect brought by the inoculation of Mycobacterium sp., that is, this strain indeed accelerated the dissipation of PAHs compared with CK soil (Fig. 2). The important mechanisms involved might be that Mycobacterium sp. synthetized dioxygenases or monooxygenases which was able to degrade PAHs. It is known that bacterial dioxygenases can catalyze the incorporation of both atoms of oxygen and two electrons from NADH to form cisdihydrodiol, while monooxygenases can hydrolyze PAHs into trans-dihydrodiols and this activity is slower than dioxygenases (Dudhagara and Dave 2018). In addition, some researchers suggested that the particular cell wall layer of mycobacteria, such as mycolic acids, is also important for the degradation of hydrophobic compounds (Zeng et al. 2010).

\section{Conclusions}

The growth of ryegrass and inoculation of Mycobacterium sp. both accelerated the removal of PAHs, especially at an early stage (before 20 days). The inoculation of Mycobacterium sp. combined with ryegrass plantation in polluted soil was more efficient compared to ryegrass plantation alone. The assessment of PAH bioavailability by Tenax-TA extraction indicated bioavailability varied between different PAHs. For 3-4 ring PAHs, bioavailability of PAHs declined over time, while more toxic 5-ring PAH could still increase in bioavailability after 40 days. Generally, the rhizosphere effect of ryegrass and inoculation of Mycobacterium sp. did not significantly change the bioavailability of PAHs in soil. The analysis of bacterial community structure showed that the phylogenetic groups containing the potential PAH degraders were present in abundance. Overall, the ryegrass rhizosphere effect and Mycobacterium sp. bioaugmentation were both efficient in accelerating PAH removal.

Acknowledgments This work was supported by the National Water Pollution Control and Treatment Science and Technology Major Project in China (Contract 2017ZX07401002).

\section{Declarations}

Conflict of interest The authors declare that they have no conflict of interest.
Open Access This article is licensed under a Creative Commons Attribution 4.0 International License, which permits use, sharing, adaptation, distribution and reproduction in any medium or format, as long as you give appropriate credit to the original author(s) and the source, provide a link to the Creative Commons licence, and indicate if changes were made. The images or other third party material in this article are included in the article's Creative Commons licence, unless indicated otherwise in a credit line to the material. If material is not included in the article's Creative Commons licence and your intended use is not permitted by statutory regulation or exceeds the permitted use, you will need to obtain permission directly from the copyright holder. To view a copy of this licence, visit http://creativecommons.org/licenses/by/4.0/.

\section{References}

Aziz F, Syed JH, Malik RN, Katsoyiannis A, Mahmood A, Li J, Zhang G, Jones KC (2014) Occurrence of polycyclic aromatic hydrocarbons in the Soan River, Pakistan: insights into distribution, composition, sources and ecological risk assessment. Ecotoxicol Environ Saf 109: 77-84. https://doi.org/10.1016/j.ecoenv.2014.07.022

Barillot CDC, Sarde CO, Bert V, Tarnaud E, Cochet N (2013) A standardized method for the sampling of rhizosphere and rhizoplan soil bacteria associated to a herbaceous root system. Ann Microbiol 63(2):471-476. https://doi.org/10.1007/s13213-012-0491-y

Bisht S, Kumar V, Kumar M, Sharma S (2014) Innoculant technology in Populus deltoides rhizosphere for effective bioremediation of Polyaromatic hydrocarbons (PAHs) in contaminated soil, northern India. Emir J Food Agr 23(1):786-799. https://doi.org/10.9755/ejfa. v26i9. 18436

Bisht S, Pandey P, Bhargava B, Sharma S, Kumar V, Sharma KD (2015) Bioremediation of polyaromatic hydrocarbons (PAHs) using rhizosphere technology. Braz J Microbiol 46(1):7-21. https://doi.org/10. 1590/S1517-838246120131354

Bourceret A, Leyval C, de Fouquet C, Cebron A (2015) Mapping the centimeter-scale spatial variability of PAHs and microbial populations in the rhizosphere of two plants. PLoS One 10(11):1-22. https://doi.org/10.1371/journal.pone.0142851

Chen F, Tan M, Ma J, Zhang S, Li G, Qu J (2016) Efficient remediation of PAH-metal co-contaminated soil using microbial-plant combination: a greenhouse study. J Hazard Mater 302:250-261. https://doi. org/10.1016/j.jhazmat.2015.09.068

Corgi'e SC, Fons F, Beguiristain T, Leyval C (2006) Biodegradation of phenanthrene, spatial distribution of bacterial populations and dioxygenase expression in the mycorrhizosphere of Lolium perenne inoculated with Glomus mosseae. Mycorrhiza 16(3):207-212. https://doi.org/10.1007/s00572-006-0049-6

Corgi'e SC, Joner EJ, Leyvall C (2003) Rhizospheric degradation of phenanthrene is a function of proximity to roots. Plant Soil 257: 143-150. https://doi.org/10.1023/A:1026278424871

DeBruyn JM, Sayler GS (2009) Microbial community structure and biodegradation activity of particle-associated bacteria in a coal tar contaminated creek. Environ Sci Technol 43:3047-3053. https://doi. org/10.1021/es803373y

Duan Y, Shen G, Tao S, Hong J, Chen Y, Xue M, Li T, Su S, Shen H, Fu X, Meng Q, Zhang J, Zhang B, Han X, Song K (2015) Characteristics of polycyclic aromatic hydrocarbons in agricultural soils at a typical coke production base in Shanxi, China. Chemosphere 127:64-69. https://doi.org/10.1016/j.chemosphere. 2014.12.075

Dudhagara DR, Dave BP, 2018. Mycobacterium as polycyclic aromatic hydrocarbons (PAHs) degrader[M]// Mycobacterium - Research and Development. https://doi.org/10.5772/intechopen.73546 
Ferradji FZ, Mnif S, Badis A, Rebbani S, Fodil D, Eddouaouda K, Sayadi S (2014) Naphthalene and crude oil degradation by biosurfactant producing Streptomyces spp. isolated from Mitidja plain soil (north of Algeria). Int Biodeterior Biodegradation 86:300-308. https://doi. org/10.1016/j.ibiod.2013.10.003

Fu D, Teng Y, Shen Y, Sun M, Tu C, Luo Y, Li Z, Christie P (2012) Dissipation of polycyclic aromatic hydrocarbons and microbial activity in a field soil planted with perennial ryegrass. Front Environ Sci Eng 6(3):330-335. https://doi.org/10.1007/s11783-011-0366-7

Gentry T, Rensing C, Pepper IAN (2004) New approaches for bioaugmentation as a remediation technology. Crit Rev Env Sci Tec 34(5): 447-494. https://doi.org/10.1080/10643380490452362

Guo M, Gong Z, Allinson G, Tai P, Miao R, Li X, Jia C, Zhuang J (2016) Variations in the bioavailability of polycyclic aromatic hydrocarbons in industrial and agricultural soils after bioremediation. Chemosphere 144:1513-1520. https://doi.org/10.1016/j. chemosphere.2015.10.027

He Y, Chi J (2015) Phytoremediation of sediments polluted with phenanthrene and pyrene by four submerged aquatic plants. J Soils Sediments 16(1):309-317. https://doi.org/10.1007/s11368-0151221-4

Hou J, Liu W, Wang B, Wang Q, Luo Y, Franks AE (2015) PGPR enhanced phytoremediation of petroleum contaminated soil and rhizosphere microbial community response. Chemosphere 138:592598. https://doi.org/10.1016/j.chemosphere.2015.07.025

Joner EJ, Johansen A, Loibner AP, Cruz MAD, Szolar OHJ, Portal J-M, Leyval C (2001) Rhizosphere effects on microbial community structure and dissipation and toxicity of polycyclic aromatic hydrocarbons (PAHs) in spiked soil. Environ Sci Technol 35(13):27732777. https://doi.org/10.1021/es000288s

Kanaly RA, Harayama S (2010) Advances in the field of high-molecularweight polycyclic aromatic hydrocarbon biodegradation by bacteria. Microb Biotechnol 3(2):1-29. https://doi.org/10.1111/j.1751-7915. 2009.00130.x

Khanna P, Goyal D, Khanna S (2011) Pyrene degradation by Bacillus pumilus isolated from crude oil contaminated soil. Polycycl Aromat Comp 31(1):1-15. https://doi.org/10.1080/10406638.2010.542792

Lily MK, Bahuguna A, Dangwal K, Garg V (2010) Optimization of an inducible, chromosomally encoded benzo[a]pyrene $(\mathrm{BaP})$ degradation pathway in Bacillus subtilis BMT4i (MTCC 9447). Ann Microbiol 60(1):51-58

Liu R, Xiao N, Wei S, Zhao L, An J (2014) Rhizosphere effects of PAHcontaminated soil phytoremediation using a special plant named fire Phoenix. Sci Total Environ 473-474:350-358. https://doi.org/10. 1016/j.scitotenv.2013.12.027

Maiti A, Das S, Bhattacharyya N (2012) Bioremediation of high molecular weight polycyclic aromatic hydrocarbons by Bacillus thuringiensis strain NA2. J Sci 1(4):72-75

Maliszewska-Kordybach B (1996) Polycyclic aromatic hydrocarbons in agricultural soils in Poland: preliminary proposals for criteria to evaluate the level of soil contamination. Appl Geochem 11(2): 121-127. https://doi.org/10.1016/0883-2927(95)00076-3

Parrish ZD, Banks MK, Schwab AP (2005) Effect of root death and decay on dissipation of polycyclic aromatic hydrocarbons in the rhizosphere of yellow sweet clover and tall fescue. Bioremediation and Biodegradation 34:207-216. https://doi.org/10.2134/jeq2005.0207

Peng A, Liu J, Ling W, Chen Z, Gao Y (2015) Diversity and distribution of $16 \mathrm{~S}$ rRNA and phenol monooxygenase genes in the rhizosphere and endophytic bacteria isolated from PAH-contaminated sites. Sci Rep 5:1-12. https://doi.org/10.1038/srep12173

Peng C, Wang M, Zhao Y, Chen W (2016) Distribution and risks of polycyclic aromatic hydrocarbons in suburban and rural soils of Beijing with various land uses. Environ Monit Assess 188(3):162. https://doi.org/10.1007/s10661-016-5156-Z
Peng RH, Xiong AS, Xue Y, Fu XY, Gao F, Zhao W, Tian YS, Yao QH (2008) Microbial biodegradation of polyaromatic hydrocarbons. FEMS Microbiol Rev 32(6):927-955. https://doi.org/10.1111/j. 1574-6976.2008.00127.x

Pritchina O, Ely C, Smets BF (2011) Effects of PAH-contaminated soil on rhizosphere microbial communities. Water Air Soil Poll 222(14):17-25. https://doi.org/10.1007/s11270-011-0800-2

Røberg S, Østerhus JI, Landfald B (2011) Dynamics of bacterial community exposed to hydrocarbons and oleophilic fertilizer in high-Arctic intertidal beach. Polar Biol 34(10):1455-1465. https://doi.org/10. 1007/s00300-011-1003-4

Salehi SY, Deljoo S, Harzandi AM (2015) Fluorene and phenanthrene uptake and accumulation by wheat, alfalfa and sunflower from the contaminated soil. Int J Phytorem 17(12):1145-1152. https://doi. org/10.1080/15226514.2015.1045123

Shahsavari E, Adetutu EM, Taha M, Ball AS (2015) Rhizoremediation of phenanthrene and pyrene contaminated soil using wheat. J Environ Manag 155:171-176. https://doi.org/10.1016/j.jenvman.2015.03. 027

Sipila TP, Keskinen AK, Akerman ML, Fortelius C, Haahtela K, Yrjala K (2008) High aromatic ring-cleavage diversity in birch rhizosphere: PAH treatment-specific changes of I.E.3 group extradiol dioxygenases and 16S rRNA bacterial communities in soil. ISME J 2(9):968-981. https://doi.org/10.1038/ismej.2008.50

Srogi K (2007) Monitoring of environmental exposure to polycyclic aromatic hydrocarbons: a review. Environ Chem Lett 5:169-195. https://doi.org/10.1007/s10311-007-0095-0

Tejeda-Agredano MC, Gallego S, Vila J, Grifoll M, Ortega-Calvo JJ, Cantos M (2013) Influence of the sunflower rhizosphere on the biodegradation of PAHs in soil. Soil Biol Biochem 57:830-840. https://doi.org/10.1016/j.soilbio.2012.08.008

Tyagi M, da Fonseca MM, de Carvalho CC (2011) Bioaugmentation and biostimulation strategies to improve the effectiveness of bioremediation processes. Biodegradation 22(2):231-241. https://doi.org/10. 1007/s10532-010-9394-4

Uyttebroek M, Ortega-Calvo JJ, Breugelmans P, Springael D (2006) Comparison of mineralization of solid-sorbed phenanthrene by polycyclic aromatic hydrocarbon (PAH)-degrading Mycobacterium spp. and Sphingomonas spp. Appl Microbiol Biotechnol 72(4):829-836. https://doi.org/10.1007/s00253-0060337-2

Wang K, Huang H, Zhu Z, Li T, He Z, Yang X, Alva A (2013) Phytoextraction of metals and rhizoremediation of PAHs in cocontaminated soil by co-planting of Sedum alfredii with ryegrass (Lolium perenne) or castor (Ricinus communis). Int J Phytorem 15(3):283-298. https://doi.org/10.1080/15226514.2012.694501

Wu J, Gong Z, Zheng L, Yi Y, Jin J, Li X, Li P (2010) Removal of high concentrations of polycyclic aromatic hydrocarbons from contaminated soil by biodiesel. Front Environ Sci Eng in China 4(4):387394. https://doi.org/10.1007/s11783-010-0269-Z

Xu Y, Sun GD, Jin JH, Liu Y, Luo M, Zhong ZP, Liu ZP (2014) Successful bioremediation of an aged and heavily contaminated soil using a microbial/plant combination strategy. J Hazard Mater 264: 430-438. https://doi.org/10.1016/j.jhazmat.2013.10.071

Zeng J, Lin X, Zhang J, Li XZ (2010) Isolation of polycyclic aromatic hydrocarbons (PAHs)-degrading Mycobacterium spp. and the degradation in soil. J Hazard Mater 183(1-3):718-723. https://doi.org/ 10.1016/j.jhazmat.2010.07.085

Publisher's note Springer Nature remains neutral with regard to jurisdictional claims in published maps and institutional affiliations. 